\title{
Estimation accuracy vs. engineering significance of contact parameters for solid dampers
}

\author{
Original article \\ Article history: \\ Accepted: 13 April 2017 \\ Published: 4 July 2017 \\ This paper is the updated version of a \\ paper originally presented at the 1st \\ Global Power and Propulsion Forum, \\ GPPF 2017, in Zurich, Jan 16-18 2017
}

\section{D) Check for updates}

*Correspondence:

CG: chiara.gastaldiapolito.it

Peer review:

Single blind

\section{Copyright:}

(c) 2017 Gastaldi and Gola. () This is an open access article distributed under the Creative Commons Attribution Non Commercial License (CC BY-NC 4.0), which permits unrestricted use, distribution, and reproduction in any medium for noncommercial purposes only, provided the original work is properly cited and its authors credited.

\section{Keywords:}

underplatform damper; contact parameters; friction damping; harmonic balance method

\section{Citation:}

Gastaldi C., and Gola M. M. (2017). Estimation accuracy vs. engineering significance of contact parameters for solid dampers. Journal of the Global Power and Propulsion Society. 1: 71-83.

https://doi.org/10.22261/VLXC9F
Chiara Gastaldi, Muzio M. Gola

Politecnico di Torino, Corso Duca degli Abruzzi 24, DIMEAS, 10129

Torino, Italy

\begin{abstract}
All numerical models of friction-damped bladed arrays require knowledge or information of contact-friction parameters. In the literature, these parameters are typically tuned so that the experimental Frequency Response Function (FRF) of a damped blade matches its numerical counterpart. It is well known that there exist multiple combinations of contact parameters capable of satisfying a given experimental-numerical FRF match. A better approach towards a finer tuning could be based on directly measuring contact forces transmitted between blade platforms through the damper: in this case friction coefficients are estimated through tangential over normal force components during those hysteresis segments which are safely identified as being in a slip condition. This has been applied by these authors to rigid bar (solid) dampers. Unfortunately, the four contact stiffness values (left and right damper-platform contact, normal and tangential) are more than the measurements available in the technique presented by these authors. Therefore, the problem is underdetermined. The purpose of this paper is twofold, i.e., to propose an alternative way to estimate contact stiffness values (i.e. thus solving the under-determinacy mentioned above) and to check the effective significance of such estimates from a practical engineering point of view. The contact parameter estimation technique proposed by these authors produces, for each contact parameter, a best-fit value and an uncertainty band. It will be shown that the uncertainty affecting each contact parameter results in an uncertainty on the equivalent damping and stiffness indicators at blade level which is lower than $5 \%$.
\end{abstract}

\section{Introduction}

Friction damping devices are commonly used in turbines to reduce the vibration amplitude of blades in resonant conditions (Berruti and Maschio, 2012). One of these devices is the underplatform damper (UPD). The UPD is a small, cheap, metal component positioned at the underside of two adjacent blades (Srinvasan and Cutts, 1983). UPDs can come in different shapes (cylindrical, wedge or asymmetrical). As already pointed out by Panning et al. (2004) and Petrov and Ewins (2007), the asymmetrical configuration is preferred since, unlike cylindrical dampers, it avoids rolling (loss of stiffness 
and lower dissipation) and, unlike wedge dampers, it self-adapts its position so that the contact with the platforms is ensured even for large platform displacements.

The presence of UPDs serves a double purpose: (1) it introduces additional stiffness, thus shifting the resonance frequency of the damper-blades system (2) the relative motion between the damper and the platforms causes energy dissipation through friction, thus introducing additional damping.

Nevertheless, the UPD contribution to stiffness and damping is not easy to predict. To this purpose, a complex hierarchy of numerical techniques has been developed in the last two decades (Petrov and Ewins, 2003; Charleux et al., 2006; Petrov and Ewins, 2007; Cigeroglu et al., 2009; Firrone et al., 2009; Siewert et al., 2009). However, whatever the numerical technique, a solution simultaneously correct for the damper and the blade dynamics is found if and only if their interface forces are correctly reproduced.

Trustworthy predictions of contact forces are largely dependent on the appropriate choice of contact parameters (i.e. friction coefficients and contact stiffness values), as demonstrated by Berruti et al. (2007) and Schwingshackl et al. (2012). The need for the safe determination of contact parameters led to AERMEC's Test Rigs. Some focus on single contacts under constant normal loads and high temperatures (Lavella et al., 2011; Botto et al., 2012; Lavella, 2016), while the Damper Test Rig, built in 2008 (Gola et al., 2010) aims at estimating contact parameters of UPDs under more realistic working conditions in terms of variable normal load and complex interface kinematics. Since then, the test rig has been used to investigate the behaviour of several dampers in terms of kinematics and force transmission characteristics (Gola and Liu, 2014). Subsequent improvements have been performed on the test rig structure, to increase its operating frequency range (Gola et al., 2012) (now up to $160 \mathrm{~Hz}$ ) and the contact pressure on the damper (Gastaldi and Gola, 2016a) (up to $6 \mathrm{MPa}$ for plane-on-plane contacts).

A numerical model of the damper/test-rig system was presented by Gola and Liu (2014), together with the first version of the contact parameters estimation procedure. This procedure was successful in estimating friction coefficients, however the contact stiffness problem was left underdetermined.

The first section of the paper overcomes this limitation, thus identifying a unique value for each of the four contact stiffness - left and right damper-platform contact, normal and tangential. In it, new experimental capabilities and data processing techniques are added to the measurement protocol developed by these authors (Gola and Liu, 2014). Specifically, platform-to-damper measurements of tangential force vs. relative tangential displacement (platform-to-damper hysteresis cycles), left and right, first presented in Gastaldi and Gola, 2016b, are here improved and analysed. Tangential contact stiffness values are directly the slopes of those hysteresis segments which are safely identified as being in a stick condition. In flat-on-flat contacts which are found in solid dampers, normal contact stiffness values cannot be measured directly. This paper proposes to deduce them by plotting the damper-platform relative rotation vs. the contact force eccentricity, introducing a novel formulation which overcomes the limitations found in Gastaldi and Gola, 2016b. By these means all contact parameters are determined for a solid damper purposely designed to avoid undesirable lift-off and to allow accurate experimental conditions (Gastaldi and Gola, 2016a).

Uncertainty estimates are also shown. The section is closed by feeding the contact parameters into a numerical model representing the damper in the test rig including the rig's own pertinent spring values: on this basis, a numerically simulated platform-to-platform cycle is compared against its experimental counterpart.

The second section of the paper numerically investigates the influence of variations of each damper-toplatform contact parameters on those platform-to-platform blade coupling factors which are of a major relevance to engineering applications. The following have been chosen: the real and the imaginary parts of the coupling stiffness, i.e. the equivalent spring and damping values. The sensitivity of each contact parameter can thus be compared against its experimental accuracy from the first section. 


\section{The test rig}

The test rig (see Figure 1a), developed over the years by the AERMEC laboratory, is designed aiming at:

- imposing user-defined in-plane harmonic displacements simulating the so-called In-Phase (vertical) and Out-of-Phase (horizontal) relative motion between the blades platforms or combinations of the two;

- measuring the forces transmitted between the two platforms through the damper;

- measuring the damper in-plane kinematics.

To achieve the first goal the left platform is connected to two piezoelectric actuators. To achieve the second goal the right platform is connected to two uniaxial force sensors by means of a tripod and the damper is pulled by a deadweight simulating the centrifugal force. The third goal is achieved by utilizing a differential laser head to measure the platforms relative displacement (a necessary precaution owing to the lack of closed loop control of the piezoelectric actuators) and the damper radial displacement and rotation angle. A complete description of the test rig and of the measurement protocol can be found in Gola and Liu, 2014.

The ultimate goal of the test rig is to relate the imposed displacement (1) to the contact forces (2) in order to record the damper platform-to-platform hysteresis loop (as shown in Figure 2) and link its different portions to the corresponding damper kinematic behaviour (3). The area of the global hysteresis cycle represents the energy dissipated by the damper.

\section{The numerical model}

Figure $1 \mathrm{~b}$ shows the numerical model of the damper/platform system. In it, the damper is modelled as a rigid body with mass and inertia properties. The rigid body assumption is considered valid given the bulkiness of the damper and has been successfully applied to several test cases in the last few years (Gola and Gastaldi, 2014; Gola and Liu, 2014; Gastaldi and Gola, 2016a). The relative motion imposed to the platforms is harmonic to simulate the motion of the blades at resonance (and that of the test rig).

The contact model used is a standard macroslip element (Srinvasan and Cutts, 1983). The following section will present the procedure used to tune the contact parameters (six in total): with reference to Figure $1 \mathrm{~b}$, friction coefficients $\left(\mu_{\mathrm{R}}, \mu_{\mathrm{L}}\right)$, tangential contact stiffness $\left(\mathrm{k}_{\mathrm{tR}}, \mathrm{k}_{\mathrm{t}}\right)$ and normal contact stiffness $\left(\mathrm{k}_{\mathrm{nR}}, \mathrm{k}_{\mathrm{nL}}\right)$. The flat-on-flat contact is represented using multiple macroslip elements (typically 4 , as in the present case): the $\mathrm{k}_{\mathrm{tL}}$ and $\mathrm{k}_{\mathrm{nL}}$ values refer to the complete interface.
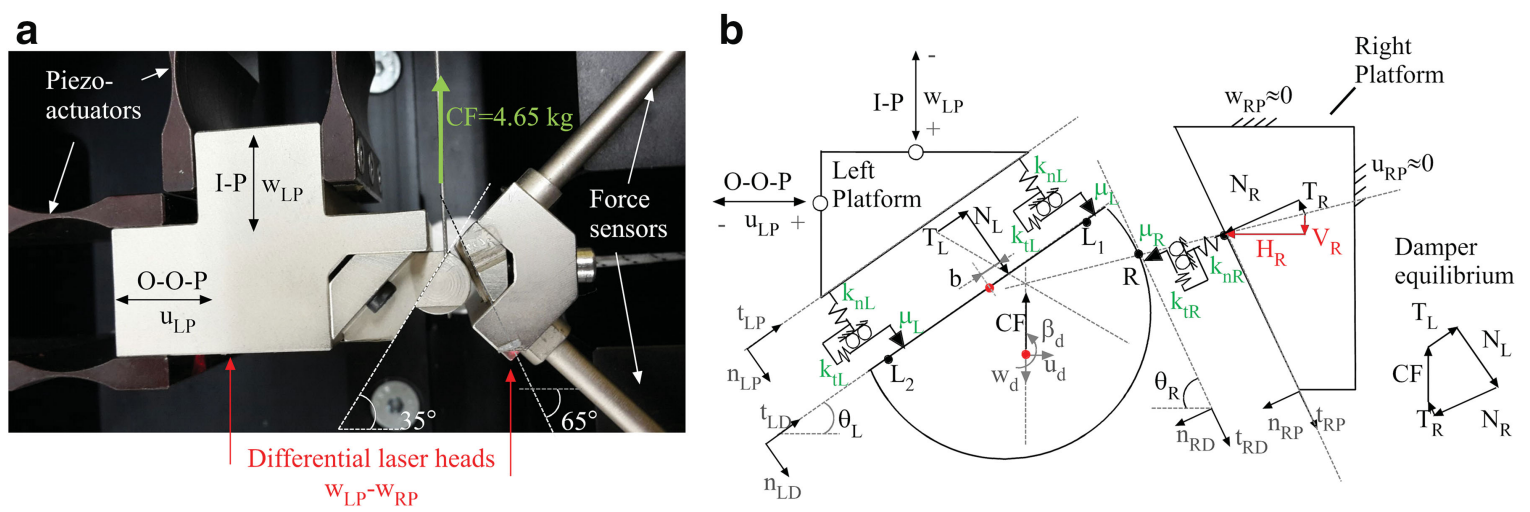

Figure 1. (a) Damper test rig experimental set-up. (b) Corresponding numerical model. 


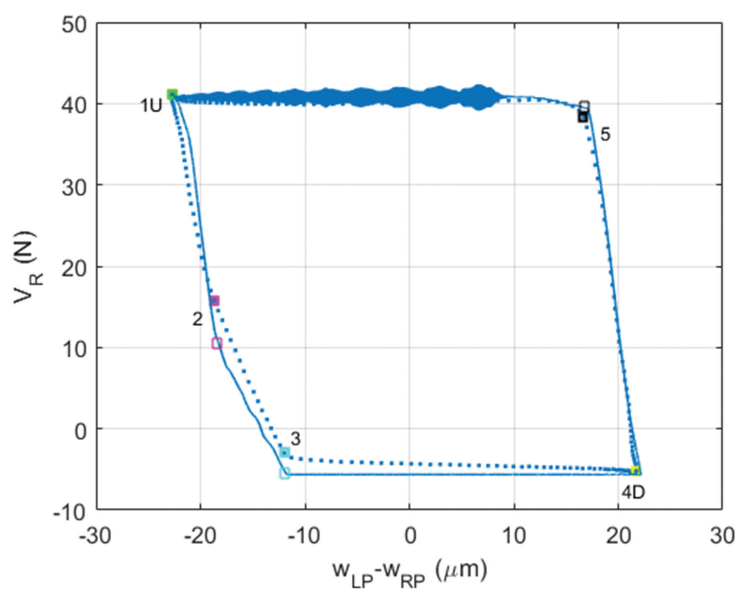

Figure 2. Measured (dotted line) and simulated (solid line) platform-to-platform In Phase (IP) hysteresis cycle for the damper in Figure $1 \mathrm{a}$.

\section{The benchmark case}

The experimental benchmark has been chosen by imposing a $\mathrm{w}_{\mathrm{LP}}=30 \mu \mathrm{m}$ (reduced to $22 \mu \mathrm{m}$ due to the lack of a closed loop control on the piezo) InPhase harmonic motion to the left platform. The centrifugal load on the damper is set at $50 \mathrm{~N}$. The frequency is set to $5 \mathrm{~Hz}$ in order to have a quasistatic hysteresis measurement (i.e. cleaner signal) for contact parameter estimation. It should be noted that the damper has been tested in the complete frequency range allowed by the test rig $([5-160 \mathrm{~Hz}])$ and the results have been found consistent, as already shown in Gola et al., 2012.

The centrifugal force is set at $50 \mathrm{~N}$. However, the contact pressure is greatly increased by the presence of two $4 \mathrm{~mm}$ long tracks on the platforms, described in Gastaldi and Gola, 2016a. These tracks have a double function: they ensure a controlled contact length and increase the contact pressure to $50 \%$ of that experienced by a $170 \mathrm{~mm}$ long damper with the same cross section mounted on the fourth stage of a power-plant turbine known to the authors.

Figure 2 plots the comparison between numerical and experimental vertical platform-to-platform hysteresis cycle. The measured cycle is reproduced very satisfactorily (error on equivalent stiffness and damping $<1 \%$ ), although the slopes visible in Figure 2 were not used for tuning the contact parameters; the same holds for contact stage transitions and force levels.

Figures 3 to 5 display additional experimental diagrams, relative to the same measurement and experimental conditions: this set of diagrams, described in the following section will be used to estimate the damper/platforms contact parameters.

\section{Estimation of contact parameters}

The following paragraphs describe the estimation procedure for each of the six contact parameters needed to calibrate the damper model. For each contact parameter a subsection is devoted to estimation accuracy. In all cases the authors have taken into account three contributions: measurement uncertainty (due to instruments uncertainty, properly propagated where needed), uncertainty introduced by data processing techniques (e.g. reading error) and finally sample-to-sample variability (repeated measurements). Depending on the specific contact parameter, one or more contributions may be negligible with respect to others.

\section{Friction coefficients}

Friction coefficients at both interfaces are estimated using the time history of the tangential/normal force ratio at the two contacts as described in Gola and Liu, 2014, and Gastaldi and Gola, 2016a. If the force ratio is constant in time and equal to a maximum then it is assumed to be a friction coefficient (e.g. 3-4D and 5-1U in Figure 3). The measured values for the case in Figure 3 are reported in Table 1.

The components of the right contact force $\left(T_{R}\right.$ and $\left.N_{R}\right)$ are directly measured, while the left components $\left(T_{L}\right.$ and $\mathrm{N}_{\mathrm{L}}$ ) are reconstructed: the numerical model has shown that inertia effects of the damper are negligible in the investigated frequency range therefore it is possible to reconstruct the equilibrium as shown in Figure 1b.

\section{Estimation accuracy}

The variability between different test runs is negligible, therefore the estimation accuracy includes two effects: the uncertainty on the force components $\left(4 \%\right.$ on $T_{R} / N_{R}$ and $8 \%$ on $T_{L} / N_{L}$ ) derived from the 


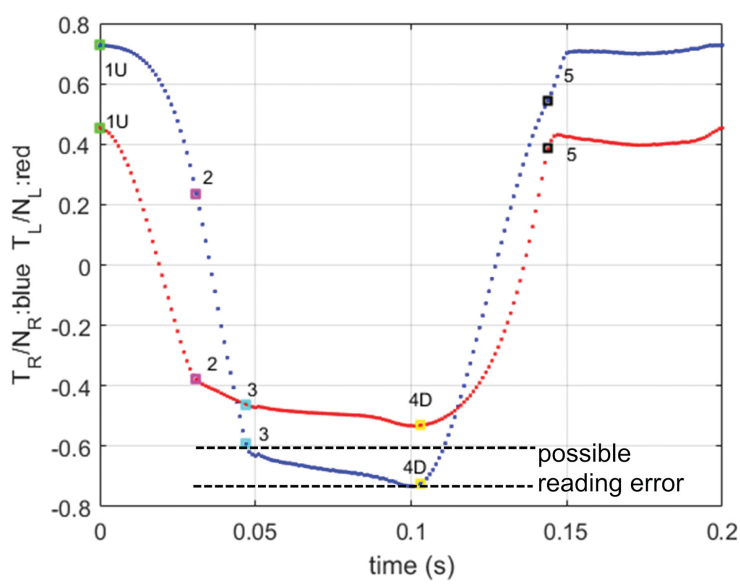

Figure 3. Measured $\mathrm{T} / \mathrm{N}$ force ratio diagram corresponding to the cycle in Figure 2. tainty). However, as it will be shown later the effect of this variation produces negligible effects on the output quantities of interest at blade level, therefore these authors still deem the model adequate.

\section{Tangential contact stiffness values}

The dummy platform have been recently equipped with cube-like protrusions oriented with one of the faces perpendicular to the contact line (see Figure 4a). Each contact line (left and right) is equipped with two cubes (one on the damper and one on the corresponding platform). This allows for the measurement of the tangential relative motion at the contact. The hysteresis at the contact (as shown in Figure $4 \mathrm{~b}$ ) is obtained by relating this relative motion to the corresponding tangential force: the slopes of the portion of hysteresis cycle identified as being in stick condition can be used to estimate the tangential contact stiffness values on each side of the damper. The stick condition is identified by looking at the corresponding T/N force ratio in Figure 3 (e.g. stage $1 \mathrm{U}-2$ is identified as stick because $\mathrm{T}_{\mathrm{L}} / \mathrm{N}_{\mathrm{L}}$ is not constant in time) as shown in (Gastaldi and Gola, 2016b). Cylinder-on-flat and flat-on-flat contact interfaces have both been tested.

\section{Estimation accuracy}

In this case measurement uncertainty (Gola and Liu, 2014; Gastaldi and Gola, 2016b) (error on dynamic variation of force summed to error on laser recorded displacement) is one order of magnitude lower with respect to the uncertainty introduced by the data processing technique. In fact, results change slightly depending on the portion of curve identified as being in stick condition. Therefore, the accuracy is here assessed by repeating the procedure on five different test runs and thus computing the standard deviation. This technique ensures that uncertainty caused by sample-to-sample variability is taken into account as well. The results are reported in Table 2.

Table 1. Accuracy and limits to ensure a $5 \%$ error on $K_{R}$ and $K_{I}$ for friction coefficients $\mu_{R}$ and $\mu_{L}$. Limits are selected as the most conservative choice encountered for $\bar{w}^{(1 C)}=[0.3-60] \mu \mathrm{m}$.

\begin{tabular}{|l|c|c|}
\hline Parameter & $\mu_{\mathrm{R}}$ & $\mu_{\mathrm{L}}$ \\
\hline Nominal value & 0.7 & 0.45 \\
\hline Experimental accuracy & {$[0.62-0.78]$} & {$[0.38-0.52]$} \\
\hline Limits to ensure $\left|\Delta K_{R}\right|<5 \%$ & {$[0.65-0.75]$} & {$[0.21-0.57]$} \\
\hline Limits to ensure $\left|\Delta K_{l}\right|<5 \%$ & {$[0.61-0.78]$} & {$[0.31-0.54]$} \\
\hline
\end{tabular}



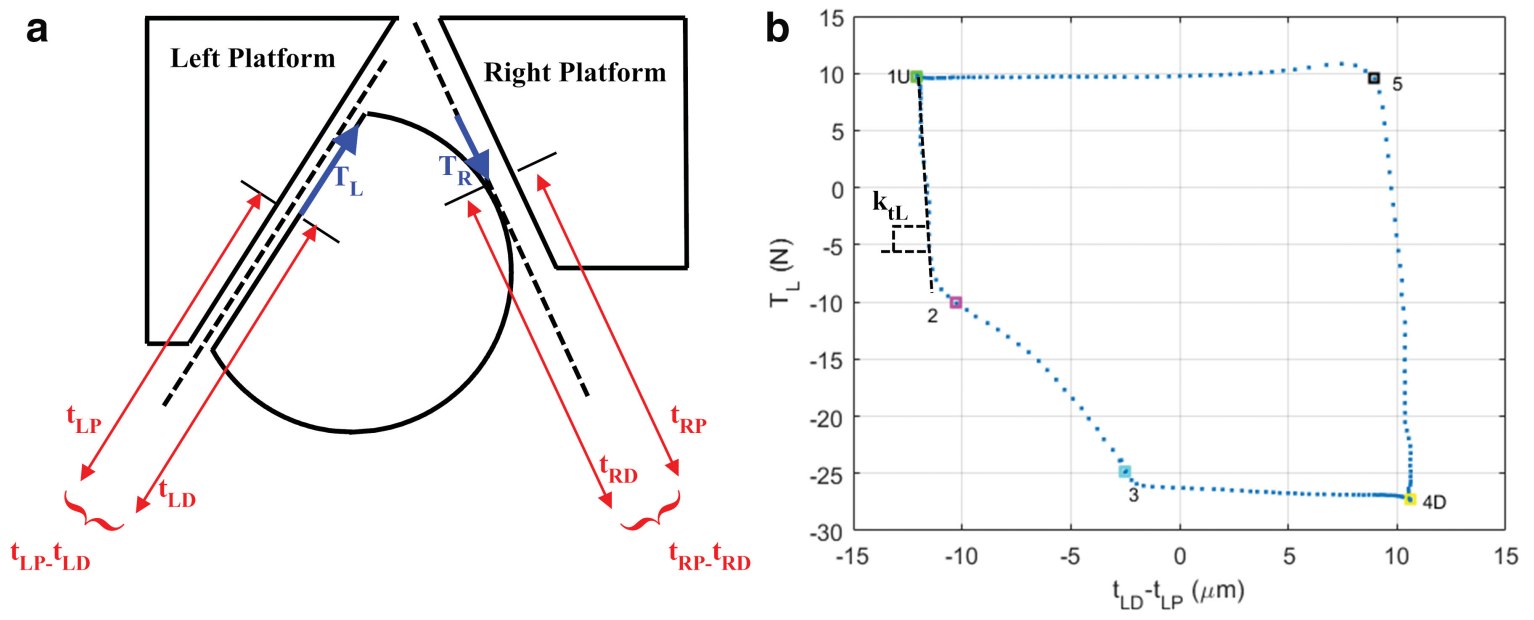

Figure 4. (a) Functional scheme representing the experimental procedure used to estimate the tangential contact stiffness values. (b) Measured platform-to-damper hysteresis at the left contact.

\section{Flat-on-flat normal contact stiffness}

The normal contact stiffness at the flat side $\mathrm{k}_{\mathrm{nL}}$ is assumed to be uniformly distributed along the flat interface, $\mathrm{dk}_{\mathrm{nL}} / \mathrm{dx}$ (see Figure 5a, (Gastaldi and Gola, 2016b)). As shown in Figure 5b during the cycle the normal component of the left contact force resultant $\mathrm{N}_{\mathrm{L}}$ travels along the flat surface during the cycle. If $\mathrm{N}_{\mathrm{L}}$ enters the inner third portion of the flat interface, then the complete surface is in contact. Under this condition, it is possible to write the moment of $\mathrm{N}_{\mathrm{L}}$ around $\mathrm{O}$ (see Figure $5 \mathrm{a}$ ) as a function of the rotation $\beta$ :

$$
\mathrm{M}=\frac{\mathrm{d} k_{n L}}{\mathrm{dx}} \beta \frac{L^{3}}{12}
$$

The normal contact stiffness per unit length is the slope highlighted in Figure $5 \mathrm{c}$. It should be noted that the slope has been estimated using a specific portion of the curve in Figure 5c: the portion corresponds to instants in the cycle where $\mathrm{N}_{\mathrm{L}}$ falls inside the inner third portion of the contact interface. This can be estimated checking the corresponding experimental diagram in Figure 5b.

\section{Estimation accuracy}

In this case measurement uncertainty (derived from instruments specs and error propagation techniques) is one order of magnitude lower with respect to the uncertainty introduced by the data

Table 2. Accuracy and limits to ensure a $5 \%$ error on $K_{R}$ and $K_{l}$ for tangential contact stiffness $k_{t R}, k_{t L}$.

\begin{tabular}{|l|c|c|}
\hline Parameter & $\mathrm{k}_{\mathrm{tR}}(\mathrm{N} / \mathrm{m})$ & $\mathrm{k}_{\mathrm{tL}}(\mathrm{N} / \mathrm{m})$ \\
\hline Nominal value & $3.0 \mathrm{e} 7$ & $2.5 \mathrm{e} 7$ \\
\hline Experimental accuracy & {$[2.8-3.2] \mathrm{e} 7$} & {$[2.1-2.9] \mathrm{e}$} \\
\hline Limits to ensure $\left|\Delta K_{R}\right|<5 \%-$ full stick state & {$[2.4-3.6] \mathrm{e} 7$} & {$[2.1-2.8] \mathrm{e}$} \\
\hline Limits to ensure $\left|\Delta K_{R}\right|<5 \%-$ gross slip state & {$[2.2-4.5] \mathrm{e} 7$} & {$[1.7-4] \mathrm{e}$} \\
\hline Limits to ensure $\left|\Delta K_{l}\right|<5 \%$ & {$[2.6-3.0] \mathrm{e} 7$} & {$[2.0-2.8] \mathrm{e}$} \\
\hline
\end{tabular}




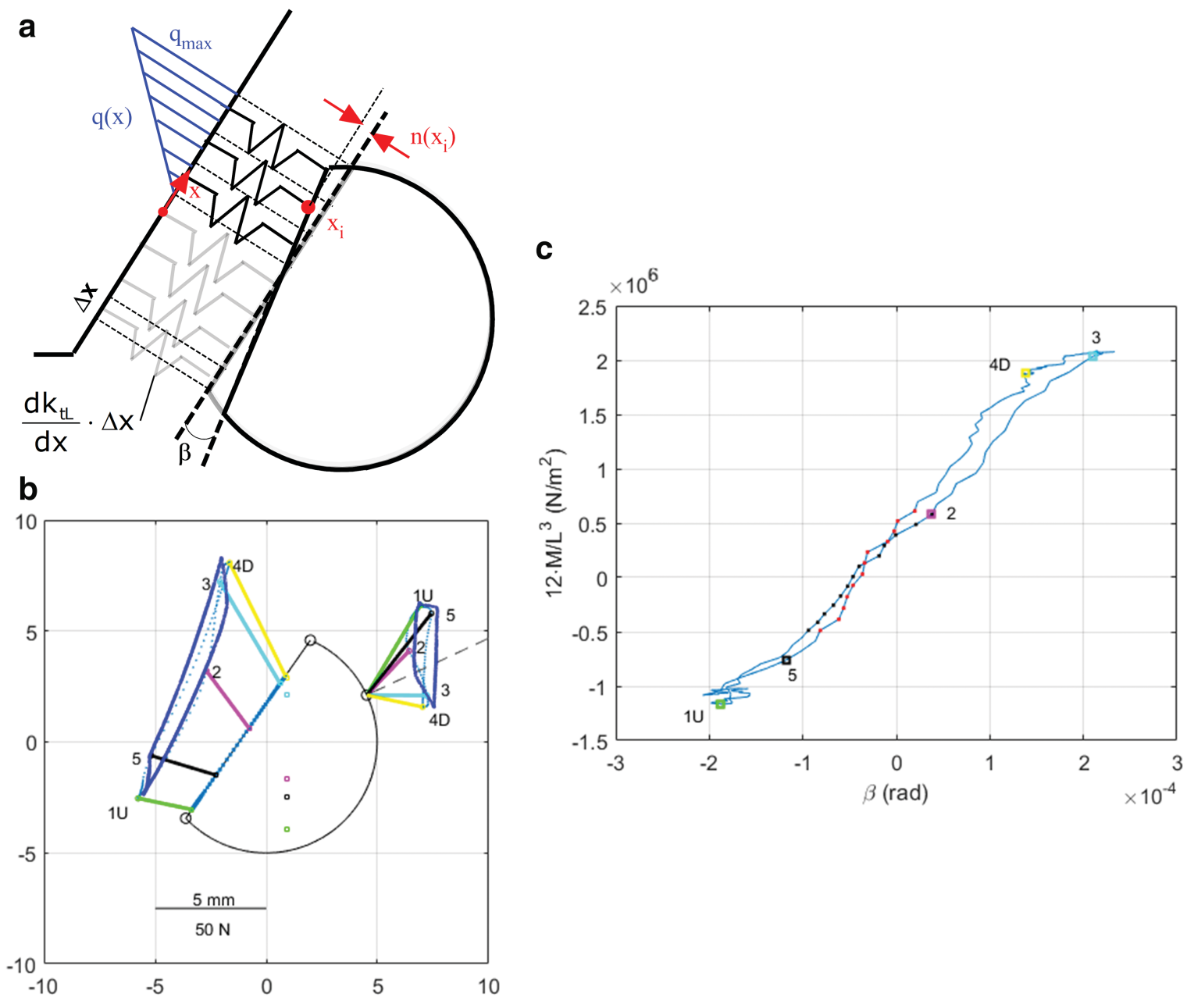

Figure 5. (a) Scheme representing the flat surface model. (b) Measured damper equilibrium during the cycle. (c) Measured moment vs. rotation plot.

processing technique. In fact, results change slightly depending on the selected portion of curve. Therefore, the accuracy is here assessed by repeating the procedure on five different test runs and thus computing the standard deviation. This technique ensures that also uncertainty caused by sample-tosample variability is taken into account. The results are reported in Table 3.

\section{Cylinder-on-flat normal contact stiffness}

The normal contact stiffness for the cylinder-on-plane contact has been obtained by taking the initial slope of the corresponding normal displacement-normal force curve. This curve has been obtained from interpolations of experimental data (Harris and Kotzalas, 2006), later confirmed by theoretical investigations (Brandlein et al., 1999).

\section{Estimation accuracy}

The value depends on the length of contact, which depends on machining tolerance (see Table 3). However, that source of uncertainty has negligible effects. The real source of uncertainty comes from the model itself (epistemic uncertainty), which may not be representative of an evolving and potentially worn contact. Thankfully it will be shown in the following sections that moderate variations of the normal contact stiffness have negligible effects $(<5 \%)$ on the equivalent stiffness and damping introduced by the damper. 
Table 3. Accuracy and limits to ensure a $5 \%$ error on $K_{R}$ and $K_{l}$ for tangential contact stiffness $k_{n R}, k_{n L}$.

\begin{tabular}{|l|c|c|}
\hline Parameter & $\mathrm{k}_{\mathrm{nR}}(\mathrm{N} / \mathrm{m})$ & $\mathrm{k}_{\mathrm{nL}}(\mathrm{N} / \mathrm{m})$ \\
\hline Nominal value & $19 \mathrm{e} 7$ & $8.4 \mathrm{e} 7$ \\
\hline Experimental accuracy & not assessed & {$[6.8-9.9] \mathrm{e} 7$} \\
\hline Limits to ensure $\left|\Delta K_{R}\right|<5 \%-$ full stick state & {$[3 e 7, ?)$} & {$[2.1-2.8] \mathrm{e}$} \\
\hline
\end{tabular}

\section{Results and discussion}

\section{Harmonic complex springs: equivalent stiffness and damping}

The nonlinear dynamic response is usually computed using Harmonic Balance methods (HBM) where forces and displacements are decomposed in their harmonic components. If the platforms' imposed displacement is assumed to be mono-harmonic $\mathrm{w}_{\mathrm{LP}}-\mathrm{w}_{\mathrm{RP}}=\overline{\mathrm{w}}^{(1 \mathrm{C})} \cos (\omega \mathrm{t})$, then it is sufficient to consider the first harmonic of the corresponding friction force $V_{R} \cong \bar{V}_{R}{ }^{(1 C)} \cos (\omega t)+i \bar{V}_{R}{ }^{(1 S)} \sin (\omega t)$ to correctly represent respectively the real and imaginary part of a complex spring $K_{R}+i K_{i}$. The real part $K_{R}=\bar{V}_{R}^{(1 C)} / \bar{w}^{(1 C)}$, in phase with the displacement, represents the elastic stiffness introduced by the damper between the platforms, the imaginary part $K_{I}=\bar{V}_{R}^{(1 S)} / \bar{w}^{(1 C)}$, out of phase with respect to the input displacement, represents the energy dissipated by the damper (i.e. the area of the hysteresis cycle equals the area of the ellipse $\left.\mathrm{A}=\pi \cdot \overline{\mathrm{w}}^{(1 \mathrm{C})} \cdot \overline{\mathrm{V}}_{\mathrm{R}}{ }^{(1 S)}\right)$. The recorded difference between the area of the hysteresis cycle and that of the corresponding ellipse is lower than $\pm 0.05 \%$, and it is due only to numerical errors in the integration. In fact, if the motion is mono-harmonic, only the corresponding component of the friction force will produce any dissipation (all others being orthogonal to the displacement), retaining multiple harmonics in the force signal will improve the shape of the resulting cycle but will not modify its area. Therefore, the HBM can be considered a simplified yet adequate representation of the original hysteresis shape (see Figure 6).

Figure 7 a plots the complex springs values against the imposed displacement $\overline{\mathrm{w}}^{(1 \mathrm{C})}$. Figure $7 \mathrm{~b}$ plots

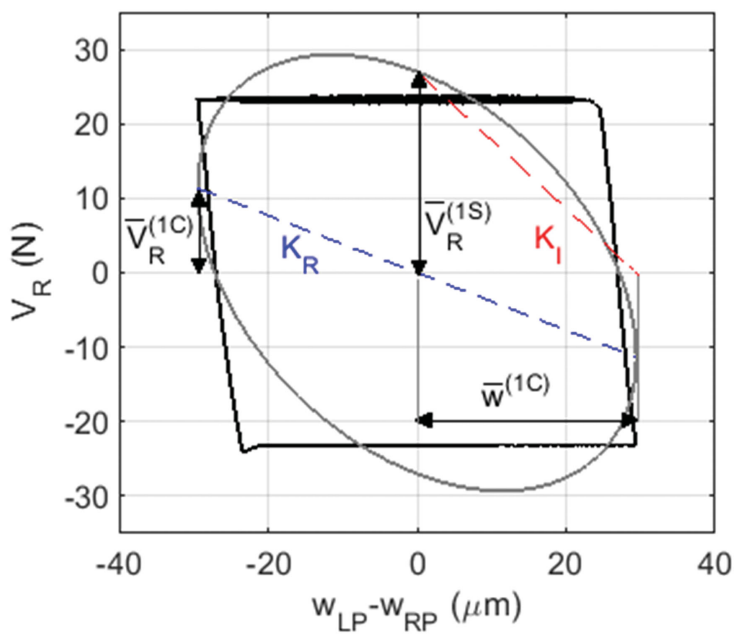

Figure 6. Hysteresis cycle with imposed displacement $\bar{W}^{(1 C)}=30 \mu \mathrm{m}$ (black line) and corresponding HBM equivalent ellipse (grey line). three hysteresis cycles corresponding to three different points highlighted on the complex springs curves. For $\overline{\mathrm{w}}^{(1 \mathrm{C})}<1 \mu m$ the damper is fully stuck to the platforms, therefore $\mathrm{K}_{1}=0$ and $\mathrm{K}_{\mathrm{R}}$ is equal to its maximum. For $\overline{\mathrm{w}}^{(1 \mathrm{C})}=5 \mu \mathrm{m}$, $\mathrm{K}_{\mathrm{I}}$ is at its maximum since $5 \mu \mathrm{m}$ is the lowest displacement that allows the damper to reach gross slip. For higher values of $\overline{\mathrm{w}}^{(1 \mathrm{C})}, \mathrm{K}_{\mathrm{I}}$ and $\mathrm{K}_{\mathrm{R}}$ progressively diminish. These complex spring values are used as performance indicators (of damper equivalent stiffness and dissipation) in the following section.

\section{Sensitivity}

The purpose of this section is to show the acceptable variation of each contact parameter to ensure an error on the performance indicators 
a

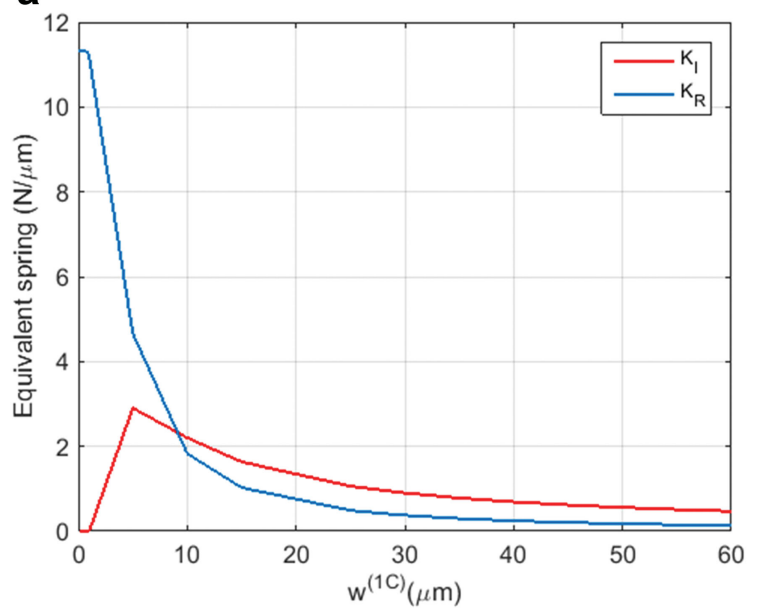

b

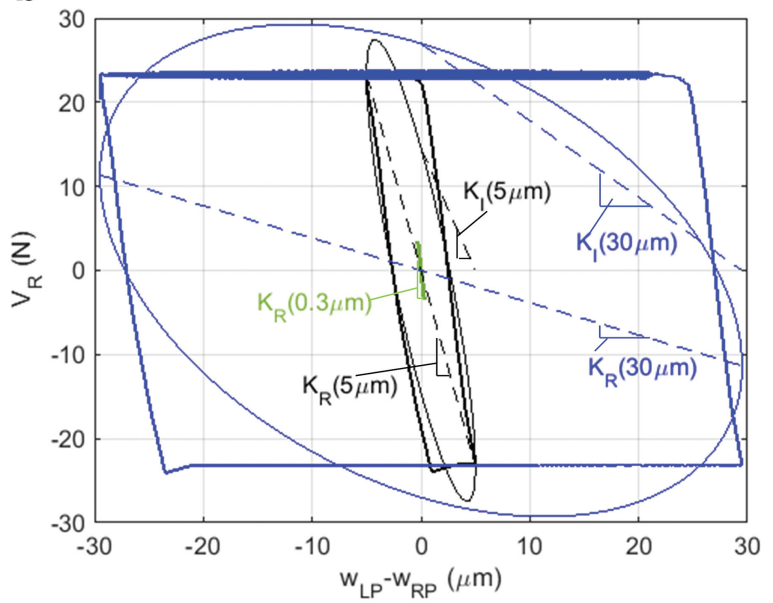

Figure 7. (a) Complex spring values against imposed displacements. (b) Corresponding platform-toplatform hysteresis cycles for increasing imposed displacements.

limited to $5 \%\left(\Delta \mathrm{K}_{\mathrm{R}}= \pm 5 \%, \Delta \mathrm{K}_{\mathrm{I}}= \pm 5 \%\right)$. The benchmark case draws the contact parameter values from the tuning procedure described in Section 3, the resulting spring values are diagrammed in Figure 7a. It was here decided to assess the effect of each contact parameter independently, i.e. starting from the benchmark case, numerically vary one at a time, while keeping the others set at their "nominal" value.

This numerical analysis is carried out for platforms' imposed displacements ranging from $\overline{\mathrm{w}}^{(1 \mathrm{C})}=0.3$ to $60 \mu \mathrm{m}$. This range of displacements has been selected because it encompasses a broad range of damper contact states (i.e. from full stick to gross slip at all interfaces). The actual working conditions of the damper will depend on the blade, the mode of vibration and the excitation amplitude, however the authors' intention was that of offering a comprehensive overview of all damper operating conditions. For each value of imposed displacement the acceptable variation of each contact parameter is compared to its accuracy.

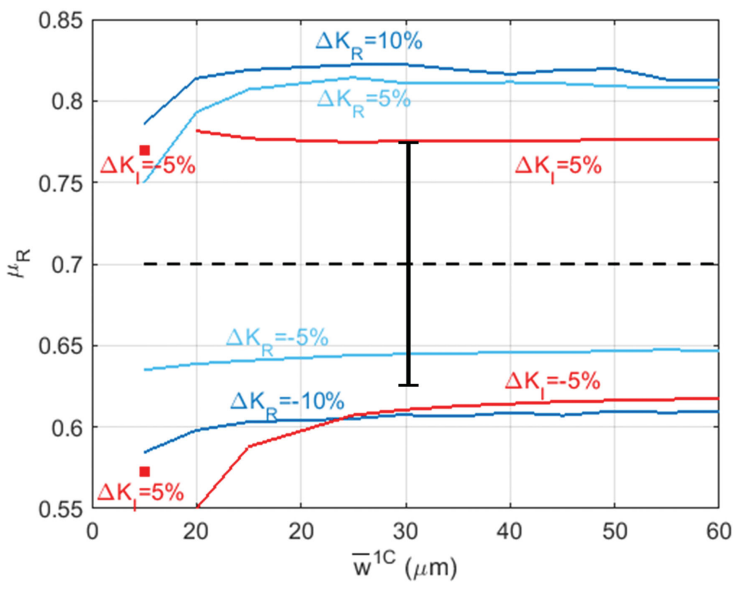

Figure 8 . Friction coefficient $\mu_{\mathrm{R}}$ acceptable variation to ensure an error on the performance indicators limited to $5 \%\left(\Delta K_{R}= \pm 5 \%, \Delta K_{I}= \pm 5 \%\right)$.

\section{Sensitivity to variation of friction coefficients}

Figure 8 plots the acceptable variation of the friction coefficient on the cylindrical side $\left(\mu_{R}\right)$. A similar trend holds for $\mu_{\mathrm{L}}$ (here not shown for brevity): the relevant data is summarized in Table 1.

In Figure 8 the black dashed line corresponds to the value of friction coefficient estimated using the procedure presented in the previous section and is assumed to be the correct one. The corresponding error bars are located at $\overline{\mathrm{w}}^{(1 \mathrm{C})}=30 \mu \mathrm{m}$.

Several caveats can be drawn from the observation of these diagrams:

- in the $\overline{\mathrm{w}}^{(1 \mathrm{C})}=[0.3-5) \mu \mathrm{m}$ range, variations of friction coefficients in the ranges shown in Figure 8 do not have any effect on $\mathrm{K}_{\mathrm{R}}$ and $\mathrm{K}_{\mathrm{I}}$ since the damper is fully stuck. 
a

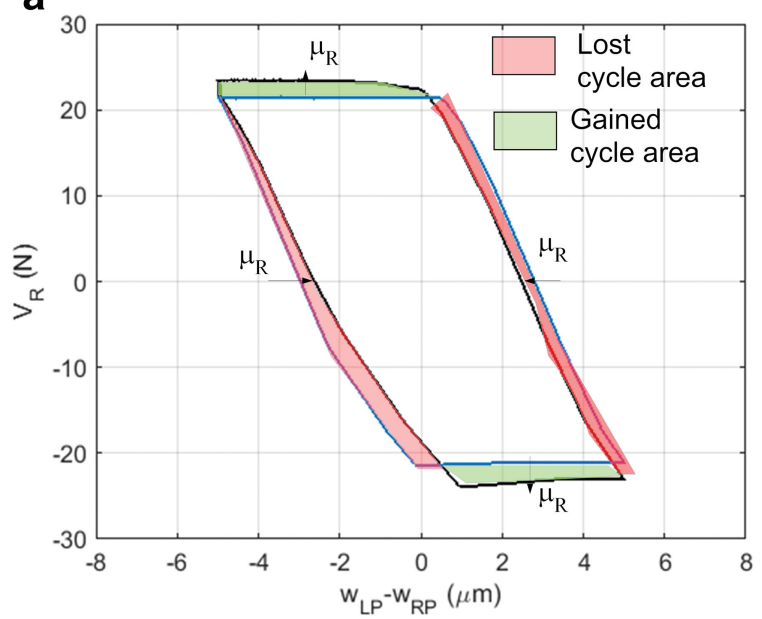

b

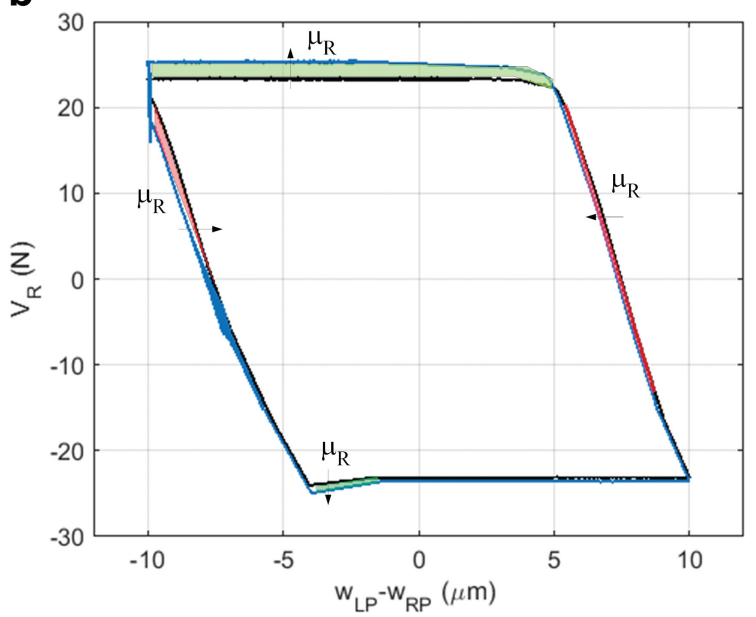

Figure 9. Effect of variation of $\mu_{R}$ on platform-to-platform hysteresis cycle area for increasing imposed displacements (a) $5 \mu \mathrm{m}$ and (b) $10 \mu \mathrm{m}$.

- in the $\overline{\mathrm{w}}^{(1 \mathrm{C})}=[5-60] \mu \mathrm{m}$ range, an increase in friction coefficient produces an increase in the limit values of the contact force $V_{R}$ and therefore an increase in $K_{R}$.

- generally speaking an increase of friction coefficient produces an increase of the dissipated energy $\left(\propto \mathrm{K}_{1}\right)$, caused by the increase of the limit values reached by the contact force $\mathrm{V}_{\mathrm{R}}$ during gross slip (see green shaded area in Figure 9b).

- when platforms' displacement is limited (in the range of $\overline{\mathrm{w}}^{(1 \mathrm{C})}=5 \mu \mathrm{m}$ ) an increase of $\mu_{\mathrm{R}}$ leads instead to a lower dissipated energy. As shown in Figure 9a, the increase of the limit values of $V_{R}$ is counter-balanced by the fact that the stick stage has a higher duration (red shaded areas in Figure 9a). The stick stage duration always increases with increasing friction coefficients, however this effect is less and less significant for increasing values of $\overline{\mathrm{w}}^{(1 \mathrm{C})}$.

- as evidenced in Table 1 the accuracy obtained with AERMEC's estimation procedure allows for a safe prediction of the equivalent spring values.

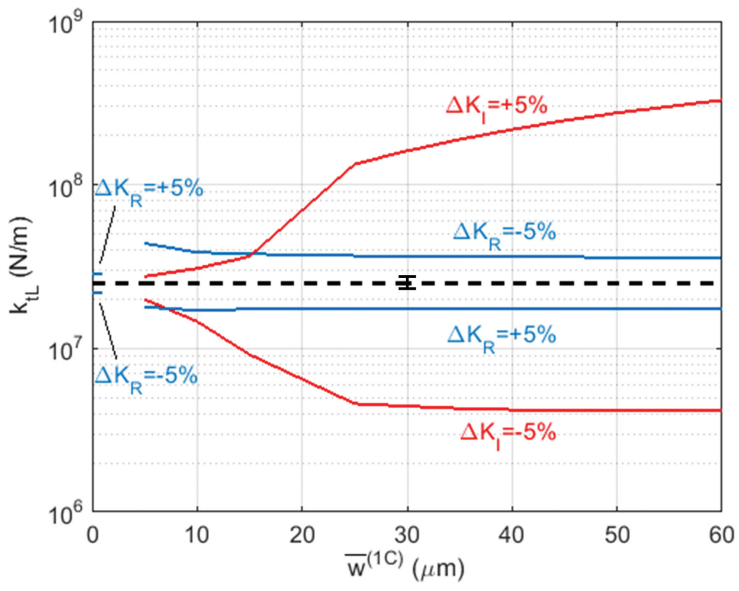

Figure 10. Tangential contact stiffness $\mathrm{k}_{\mathrm{tL}}$ acceptable variation to ensure an error on the performance indicators limited to $5 \%$ $\left(\Delta \mathrm{K}_{\mathrm{R}}= \pm 5 \%, \Delta \mathrm{K}_{\mathrm{I}}= \pm 5 \%\right)$.

\section{Sensitivity to variation of contact stiffness values}

Figure 10 plots the acceptable variation of the tangential contact stiffness at the flat side $\left(\mathrm{k}_{\mathrm{tL}}\right)$. A similar trend holds for $\mathrm{k}_{\mathrm{tR}}, \mathrm{k}_{\mathrm{nR}}$ and $\mathrm{k}_{\mathrm{nL}}$ (here not shown for brevity): the relevant data is summarized in Table 2 and Table 3. Since the limits vary with the platforms' imposed displacement the most conservative values are selected.

In Figure 10 the black dashed line corresponds to the value of contact stiffness estimated using the procedure presented in the previous section and is assumed to be the correct one. The corresponding error bars are located at $\overline{\mathrm{w}}^{(1 \mathrm{C})}=30 \mu \mathrm{m}$. 

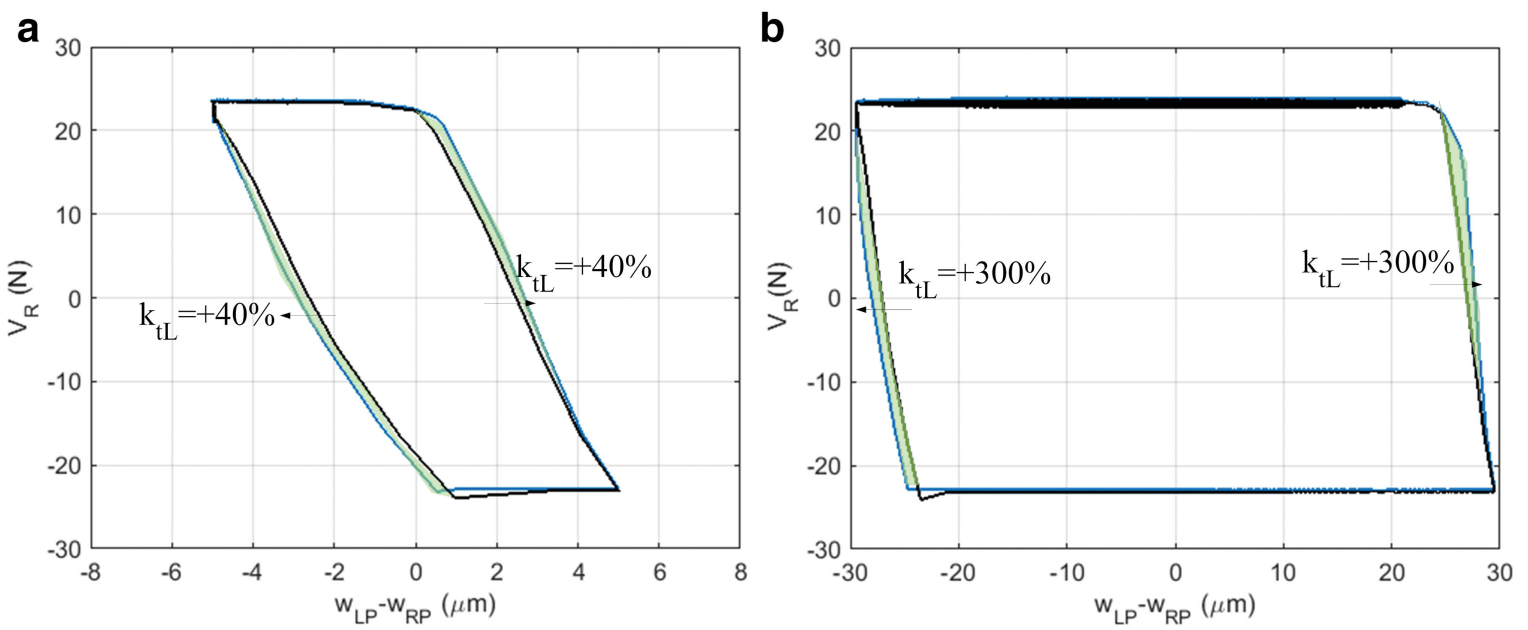

Figure 11. Effect of variation of $\mathrm{k}_{\mathrm{tL}}$ on platform-to-platform hysteresis cycle area for increasing imposed displacements (a) $5 \mu \mathrm{m}$ and (b) $30 \mu \mathrm{m}$.

Several caveats can be drawn from the observation of these diagrams:

- in the $\overline{\mathrm{w}}^{(1 \mathrm{C})}=[0.3-5) \mu \mathrm{m}$ range, only minor variations of contact stiffness values are allowed $(<15 \%)$. Since the damper is fully stuck, the cycle corresponds to a line whose slope is a linear combination of all contact stiffness values. This slope is equal to $\mathrm{K}_{\mathrm{R}}$, therefore $\Delta \mathrm{k}_{\mathrm{tL}}$ is directly proportional to $\Delta \mathrm{K}_{\mathrm{R}}$.

- in the gross slip range $\overline{\mathrm{w}}^{(1 \mathrm{C})}=[5-60] \mu \mathrm{m} \mathrm{K}_{\mathrm{R}}$ is less affected by variations of contact stiffness since the gross-slip phase prevails. Moreover, a positive $\Delta \mathrm{k}_{\mathrm{tL}}$ leads to a decrease of $\mathrm{K}_{\mathrm{R}}$. This happens because higher contact stiffness values decrease the duration of the stick phase (see Figure 10).

- in the $\overline{\mathrm{w}}^{(1 \mathrm{C})}=[0.3-5) \mu \mathrm{m}$ range, $\mathrm{K}_{\mathrm{I}}=0$ therefore the error lines cannot be plotted.

- in the range $\overline{\mathrm{w}}^{(1 \mathrm{C})}=5 \mu \mathrm{m}, \mathrm{K}_{\mathrm{I}}$ is influenced by variations of contact stiffness values because, as shown in Figure 11 the stick and gross-slip stages have a similar duration.

- in the gross slip range $\overline{\mathrm{w}}^{(1 \mathrm{C})}=[10-60] \mu \mathrm{m} \mathrm{K}_{\mathrm{I}}$ displays a diminishing sensitivity to contact stiffness values variations. This is explained by the fact that only a minimal portion of the overall area is accounted for by the variation of the hysteresis slopes (see Figure $11 \mathrm{~b}$ ). In some cases (i.e. $\mathrm{k}_{\mathrm{nR}}$, see Table 3), it was not possible to find the $\left|\Delta \mathrm{K}_{\mathrm{I}}\right|=5 \%$ limit in the investigated range.

- as evidenced in Table 2 and 3 the accuracy obtained on the tangential contact stiffness values is comparable with the $\left|\Delta \mathrm{K}_{\mathrm{R}}\right|$ and $\left|\Delta \mathrm{K}_{\mathrm{I}}\right| 5 \%$ limits.

- as evidenced in Table $3, \mathrm{k}_{\mathrm{nR}}$, being the highest, has a very low influence compared to the others.

\section{Conclusions}

The paper presents an extension of the existing contact parameter estimation technique, first described in (Gola and Liu, 2014). Specifically, a new set of experimental evidence (hysteresis cycles at the contacts and moment vs rotation diagram) was used to uniquely estimate the contact stiffness values. The contact parameters thus obtained have been fed to the numerical routine, which successfully represented the platform-to-platform hysteresis cycle.

This set of contact parameters served as a reference point to conduct a sensitivity analysis. The real and the imaginary parts of the platform-platform coupling stiffness, (i.e. the equivalent spring and damping values) have been chosen as output indicators. 
The numerical analysis has shown that the proposed contact parameters estimation technique, entirely based on direct measurements on dampers, ensures an uncertainty on the output indicators in the 5\% range.

\section{Nomenclature}

$\beta: \quad$ rotation

CF: centrifugal force on the damper

$\mathrm{H}$ : horizontal force on the damper

k: contact stiffness

$\mu$ : friction coefficient

$\mathrm{N}$ : normal force on the damper

$\mathrm{n}$ : normal displacement

$\theta$ : platform angle

$\mathrm{T}$ : tangential force on the damper

t: tangential displacement

u: horizontal displacement

$\mathrm{V}$ : vertical force on the damper

w: vertical displacement

\section{Subscripts}

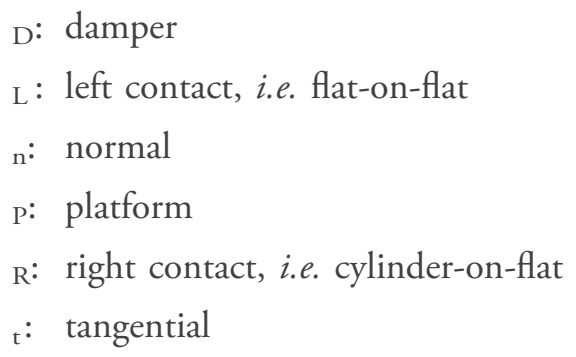

\section{Funding sources}

The Damper test rig used in this paper has been developed using funds by the DIMEAS (Politecnico di Torino Department of Mechanical and Aerospace Engineering, Italy) - AERMEC Lab research group.

\section{Competing interests}

Chiara Gastaldi declares that she has no conflict of interest. Muzio M. Gola declares that he has no conflict of interest.

\section{References}

Berruti T., Firrone C. M., Pizzolante M., and Gola M. M. (2007). Fatigue damage prevention on turbine blades: study of underplatform damper shape. Key Engineering Materials. 347: 159-164. https://doi.org/10.4028/www.scientific.net/ KEM.347.159. 
Berruti T. and Maschio V. (2012). Experimental investigation on the forced response of a dummy counter-rotating turbine stage with friction damping. Journal of Engineering for Gas Turbines and Power. 134 (12): 122502. https://doi.org/10.1115/ 1.4007325 .

Botto D., Lavella M., and Gola M. M. (2012). Measurement of contact parameters of flat on flat contact surfaces at high temperature. ASME Turbo Expo 2012: Turbine Technical Conference and Exposition. 7: 1325-1332. https://doi.org/ $10.1115 / G T 2012-69677$.

Brandlein J., Eschmann P., Hasbargen L., and Weigand K. (1999). Ball and Roller Bearings: Theory, Design and Application, 3rd Edition. New York: John Wiley and Sons. ISBN: 978-0-471-98452-8.

Charleux D., Gibert C., Thouverez F., and Dupeux J. (2006). Numerical and experimental study of friction damping in blade attachments of rotating bladed disks. International Journal of Rotating Machinery. 2006: 1-13. https://doi.org/ 10.1155/IJRM/2006/71302.

Cigeroglu E., An N., and Menq C. H. (2009). Forced response prediction of constrained and unconstrained structures coupled through frictional contacts. ASME Journal of Engineering for Gas Turbines and Power. 131 (2): 022505. https:// doi.org/10.1115/1.2940356.

Firrone C. M., Zucca S., and Gola M. M. (2009). Effect of static/dynamic coupling on the forced response of turbine bladed disks with underplatform dampers. ASME Turbo Expo 2009: Power for Land, Sea, and Air. 6: 429-440. https://doi.org/ 10.1115/GT2009-59905.

Gastaldi C. and Gola M. M. (2016a). Pre-optimization of asymmetrical underplatform dampers. Journal of Engineering for Gas Turbines and Power. 139 (1): 012504. https://doi.org/10.1115/1.403419.

Gastaldi C. and Gola M. M. (2016b). Testing, simulating and understanding under-platform damper dynamics. ECCOMAS Congress. 2016: 1-12. https://doi.org/10.7712/100016.2134.11184.

Gola M. M., Braga dos Santos M., and Liu T. (2010). Design of a new test rig to evaluate underplatform damper performance. ASME 2010 10th Biennial Conference on Engineering Systems Design and Analysis. 5: 85-94. https://doi.org/ 10.1115/ESDA2010-24268.

Gola M. M., Braga Dos Santos M., and Liu T. (2012). Measurement of the scatter of underplatform damper hysteresis cycle: experimental approach. ASME 2012 International Design Engineering Technical Conferences and Computers and Information in Engineering Conference. 1: 359-369. https://doi.org/10.1115/DETC2012-70269.

Gola M. M. and Gastaldi C. (2014). Understanding complexities in underplatform damper mechanics. ASME Turbo Expo 2014: Turbine Technical Conference and Exposition. 7A: V07AT34A002. https://doi.org/10.1115/GT2014-25240.

Gola M. M. and Liu T. (2014). A direct experimental-numerical method for investigations of a laboratory under-platform damper behavior. International Journal of Solids and Structures. 51 (25-26): 4245-4259. https://doi.org/10.1016/j. ijsolstr.2014.08.011.

Harris T. A. and Kotzalas M. N. (2006). Rolling Bearing Analysis, 5th Edition. Boca Raton, Florida: CRC Press. ISBN: 0849381673 .

Lavella M. (2016). Contact properties and wear behaviour of nickel based superalloy René 80. Metals. 6 (7): 159. https://doi. org/10.3390/met6070159.

Lavella M., Botto D., and Gola M. M. (2011). Test rig for wear and contact parameters extraction for flat-on-flat contact surfaces. ASME/STLE 2011 International Joint Tribology Conference. 1: 307-309. https://doi.org/10.1115/IJTC201161234.

Panning L., Popp K., Sextro W., Goetting F., Kayser A., and Wolter I. (2004). Asymmetrical underplatform dampers in gas turbine bladings: Theory and application. ASME Turbo Expo 2004: Power for Land, Sea, and Air. 6: 269-280. https://doi org/10.1115/GT2004-53316.

Petrov E. P. and Ewins D. (2003). Analytical formulation of friction interface elements for analysis of nonlinear multi-harmonic vibrations of bladed discs. ASME Journal of Turbomachinery. 125 (2): 364-371. https://doi.org/10.1115/1.1539868.

Petrov E. P. and Ewins D. J. (2007). Advanced modelling of underplatform friction dampers for analysis of bladed disk vibration. ASME Journal of Turbomachinery. 129 (1): 143-150. https://doi.org/10.1115/1.2372775.

Schwingshackl C. W., Petrov E. P., and Ewins D. J. (2012). Effects of contact interface parameters on vibration of turbine bladed disks with underplatform dampers. Journal of Engineering for Gas Turbines and Power. 134 (3): 032507. https://doi. org/10.1115/1.4004721

Siewert C., Panning L., Wallaschek J., and Richter C. (2009). Multiharmonic forced response analysis of a turbine blading coupled by nonlinear contact forces. ASME Turbo Expo 2009: Power for Land, Sea, and Air. 6: 231-243. https://doi.org/ 10.1115/GT2009-59201.

Srinvasan A. V. and Cutts D. G. (1983). Dry friction damping mechanisms in engine blades. Journal of Engineering for Power. 105 (2): 332-341. https://doi.org/10.1115/1.3227420. 